\title{
Hybrid Finite-Discrete Element Method Modelling of Brazilian Disc Tests
}

\author{
Huaming An ${ }^{1, \text { a }}$, Jianjun Shi ${ }^{2, b}$, Xin Zheng ${ }^{3, c}$ and Xuguang Wang ${ }^{4, d}$
}

${ }^{1}$ School of Civil \& Environmental Engineering, University of Science and Technology Beijing, China

${ }^{2}$ Key Laboratory of Ministry of Education of China for High-efficient Mining and safety of Metal Mines University of Science and Technology Beijing, China

${ }^{3}$ College of Resources \& Civil Engineering, Northeastern University, Shenyang Liaoning ,China

${ }^{4}$ Beijing General Research Institute of Mining and Metallurgy , Beijing China

a Huaming.an@yahoo.com, b keyan@ces.ustb.edu.cn, ${ }^{c}$ Xin.zheng@utas.edu.au, ${ }^{d}$ xuguang.wang@hotmail.com

Keywords: Hybrid finite-discrete element method; Brazilian disc test; combined single and smeared crack model ; Transition from continuum to discontinuum; Fracture and fragmentation

Abstract. A Hybrid finite-discrete element Method is introduced to simulate fracture and fragmentation processes of Brazilian disc test. The transition from continuum to discontinuum through fracture and fragmentation is implemented through a combined single and smeared crack model. An integrated development environment i.e. Y-2D/3D IDE, which incorporates the hybrid finite-discrete method and the combined single and smeared crack, is used to model the fracture and fragmentation process of Brazilian disc test. It is found that the proposed method, i.e. Hybrid finite-discrete element method, reproduces the processes of stress initiation and propagation, the fracture process and resultant fragments of rock in the Brazilian disc tests. The modelled failure patterns agree well with documented tests. It is concluded that the Hybrid finite-discrete method demonstrates its capability in modelling the behaviors of Brazilian disc under static loading rates.

\section{Introduction}

With the rapid development of computing power, interactive computer graphics and topological data structure, numerical methods are widely employed in researching the rock fracture process[1-4]. In general, the numerical methods available in the literature can be classified according to their hypothesis that the rock is modelled as a continuous or discontinuous material. Therefore, most popular numerical methods are continuum method, e.g. finite element method, and discontinuum method, e.g. Discrete element method. When sufficiently large scale objects are considered, a continuum method can be sufficient even if the geology includes fractures and faults[5] However, it should be noted that when the structures of interest have sizes comparable with the block size, discontinuities of individual rock must be taken into account[5]. Under this scenario, researchers turned to the discontinuum based method because a pure continuum based treatment is inappropriate. However, large-scale discrete element modelling demand a massive computational resources due to large number of particles and time-consuming contact detect procedures[6].

To overcome the limitations of continuum-based methods and discontinuum-based methods in modelling the fracture and fragmentation, i.e. transition from continuum to discontinuum, combined continuum and discontinuum methods, termed as Hybrid continuum-discontinuum models, might be a good choice for modelling rock fracture and fragments. In fact, Hybrid continuum-discontinuum models, e.g. Hybrid boundary element method-finite element method(BEM-FEM), discrete element method-finite element method ((DEM-FEM) and discrete element method-boundary element method (DEM-BEM) models, are already frequently used in rock engineering for flow and deformation problems of fractured rock[7]. It should be noted that there is no absolute advantage of one method over another, however some of the disadvantages inherent in one type can be avoided by combined continuum-discontinuum methods [8].

In this study, a Hybrid finite-discrete element method is introduced to model the rock failure and fragmentation process in the intact Brazilian disc test. The obtained results are compared with those well documented in the literature. The purpose of this study is to validate the method by reproducing the rock failure process [6]. 


\section{Hybrid finite-discrete element method}

Generally, a Hybrid finite element method consist of contact detection and contact interaction between individual bodies, deformability and transition from continuum to discontinuum, temporal integration scheme, and computational fluid dynamics[6, 9-13] Among them, the transition from continuum to discontinuum is regards as the key component which makes the hybrid finite discrete element method distinctive from many continuum-based method, e.g. finite element method, and discontinuum-base method, e.g. discrete element method. Here, only the key component is introduced in detail.

In order to simulate the transition from continuum to discontinuum through fracture and fragmentation of rock or rock-like mater, a combined single and smeared crack model is employed in Hybrid finite element method[6]. The typical stress-strain curve of geomaterials under loading is divided in two parts as shown in Fig.1a. The strain-hardening part is implemented through a standard way through the elasto-plastic constitutive law while the strain-softening part is implemented through a single crack model in which the cracks are assumed to coincide with the element edges and propagate when the critical value of Eq.1 is satisfied according to Griffith`s Failure Criterion.

$$
\mathrm{G}=2 \gamma
$$

where $G$ represents energy release rate and $\gamma$ represents the energy needed to extend crack surface by a unit area[14].

Before fracturing, the finite elements are assumed to be bonded together and separating them includes a bonding stress, which is the function of the separations. Fig.1b shows the relationship of crack distances, i.e. separations, and bonding stresses in the form of tensile and shear stresses and the relationship, i.e. functions, can be described by Eq. 2 .

$$
\sigma=\left\{\begin{array}{ccc}
{\left[2 \frac{\delta}{\delta_{p}}-\left(\frac{\delta}{\delta_{p}}\right)^{2}\right] f_{p},} & \text { if } & 0 \leq \delta \leq \delta_{p} \\
g(D) \cdot f_{p} & \text { if } & \delta_{p}<\delta<\delta_{u} \\
0 & \text { if } & \delta \geq \delta_{u}
\end{array}\right.
$$

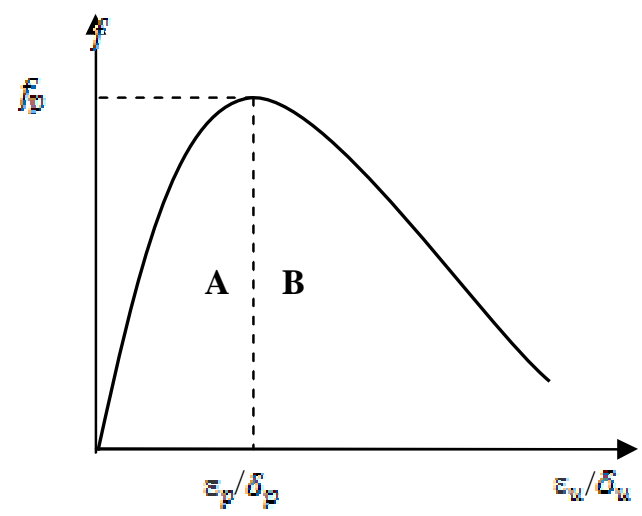

a) Stress-strain curve

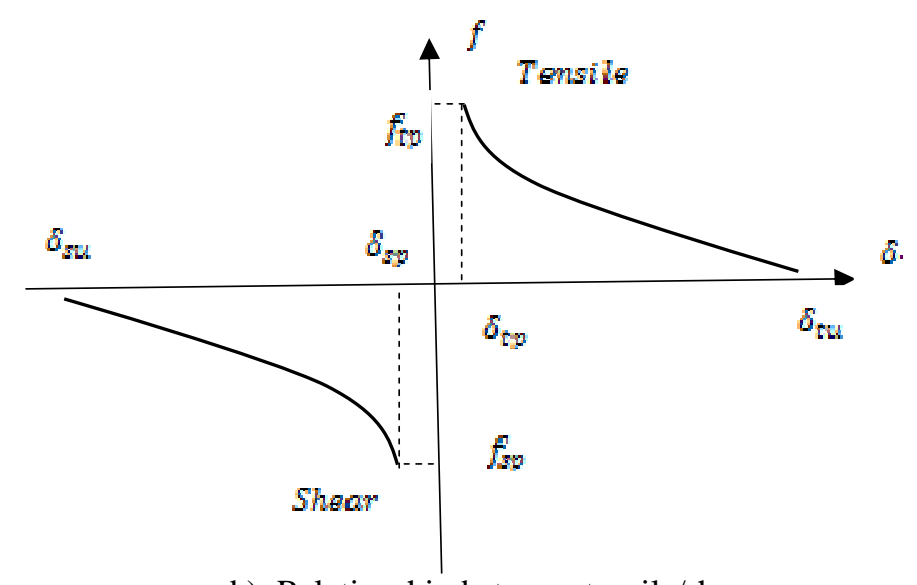

b) Relationship between tensile/shear

Fig.1. Transition from continuum to discontinuum 
where $\sigma$ is the bonding stress, $D$ is the damage index, $f_{p}$ is the peak strength, $g(D)$ is the damage function. The footnote $s$ and $t$ in Fig $1 \mathrm{~b}$ mean the variables is in terms of shear or tensile.

At separation, $\delta_{n} \geq \delta_{\mathrm{n}}$ the bonding stress becomes zero and the crack is assumed to propagate.

\section{Numerical simulation of failure and fracture process in Brazilian disc test}

A integration development environment, i.e. Y2D/3D IDE[6], implemented Hybrid finite-discrete method, is used to model the fracture and failure process of Brazilian disc test.

As shown in Fig. 2a, numerical model for the Dynamic Brazilian Disc Test is built according to the geometry suggested by ISRM, i.e. $54 \mathrm{~mm}$ in diameters of the disc. In addition to determine the tensile strength of rock mass indirectly, the cylindrical specimens is used to model the wave propagation and fracture process under various loading rates. The disc is placed between two plates with $54 \mathrm{~mm}$ in length and $3 \mathrm{~mm}$ in height. As shown in Fig. 2a the Brazilian disc test is simplified as a plane stress problems and only the vertical sections is considered. It should be noted that the loading rate, i.e. $1 \mathrm{~m} / \mathrm{s}$ on both top and bottom plates is much higher than those in laboratory static experiments. Nevertheless according to the laboratory dynamic experiment [15], the loading rates do not have important influences on rock properties until a certain threshold level is met.

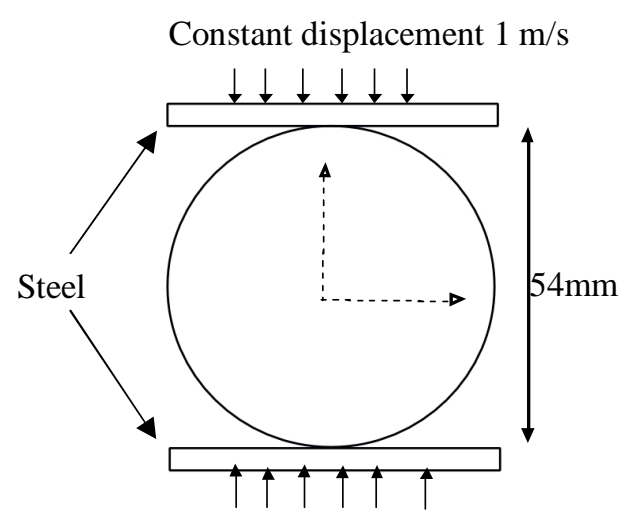

a) Geometrical model

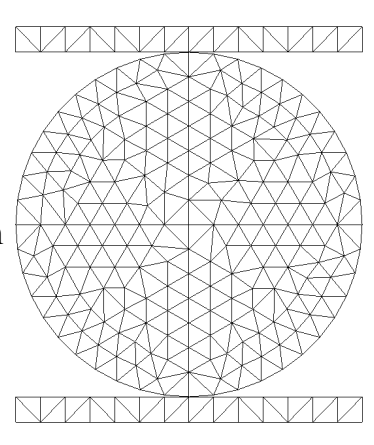

b) Numerical model

Fig. 2

Minor principal stress wave propagation. Fig. 3 depicted the modelled temporal and spatial stress wave distribution under constant displacement loading of $1 \mathrm{~m} / \mathrm{s}$. The stress distributions in the pictures correspond to the minor principal stress. It should be noted that, following the solid mechanics regulations, the compressive stresses are taken as negative, while the tensile stresses are regarded as negative. Moreover, in Fig. 3, the color represents the size of stress and the size can be referred to legend shown in Fig.3a at $1 \mu \mathbf{S}$.

As the loads are applied on top and bottom plates, almost immediately the stress waves transmit into the rock specimen without any reflection at the contact surface of plates and rock sample as shown in Fig. 3 at $1 \mu \mathrm{s}$. With time passing by from $1 \mu \mathrm{s}$ to $5 \mu \mathrm{s}$, while the stress waves are propagating upward and downwards from top and bottom contact surfaces respectively, the intensives stress begins to appear at contact surfaces area. At $5 \mu \mathrm{s}$, two stress waves from both bottom and top meet.

From $6 \mu$ s to $39 \mu$ s, the secondary stress waves, which are much stronger than the primary stress waves, continuously transmit from bottom upwards and top downwards. At $33 \mu \mathrm{s}$, the secondary stresses wave meet. In this process, the stresses are mainly distributed along the vertical diameter and they become stronger and stronger with time goes by. From $39 \mu \mathrm{s}$ to $56 \mu \mathrm{s}$, the stress waves begin to reach the disc boundary and is reflected. Consequently, the stress waves scatter in the disc. The Fig. at $168 \mu$ s is the last stress distribution pattern. Probably due to the fracture of rock and waves reflecting, there is not a clear regularities of stresses distribution. 

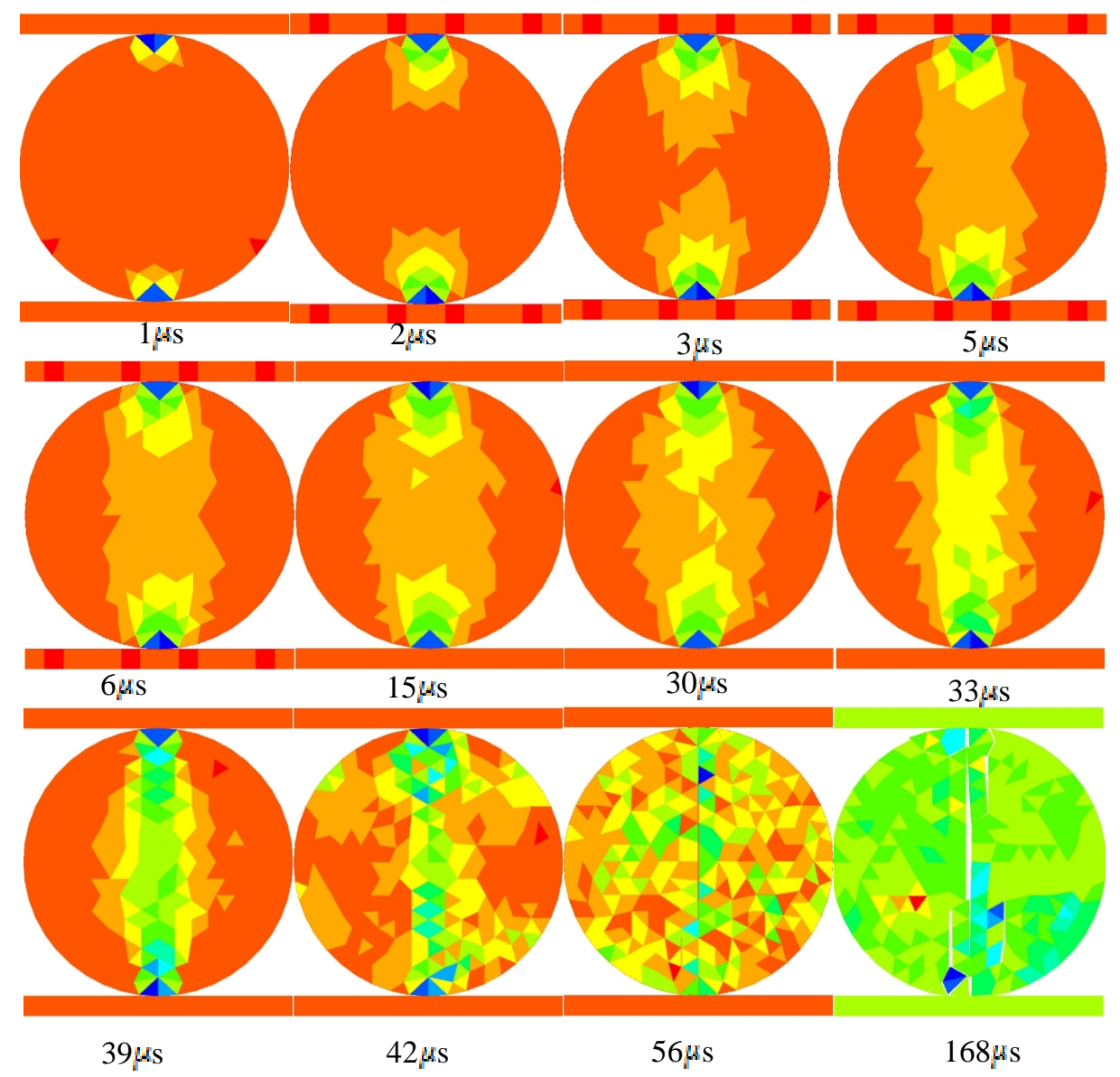

Fig .3 Minor principal stress wave propagation of rock under constant displacement increment of $1 \mathrm{~m} / \mathrm{s}$

Fracture propagation process. As shown in Fig.4, the crack initiates from the center of the loading

diameter at $34 \boldsymbol{\mu}$ s. Since the tensile stresses firstly exceed the tensile strength of the material at the center of loading diameter, the crack initiates from the center line. Then the crack propagates along the loading line to the top and bottom of the disc. These cracks are regard as primary cracking along the loading diameter[16].Afterwards, further cracks (secondary cracks) appear on both bottom and top sides of the disc at $41 \mu \mathrm{S}$ and propagate from the top and bottom of disc to the center. The secondary cracks primarily are parallel to the loading diameter. As illustrates in Fig. 3 from $42_{\mu \mathbf{S}}$ to 46 $\mu \mathrm{S}$, cracks appear on both sides of the loading diameter. Meanwhile, the primary cracks, i.e. cracks along loading diameter, continue to crack and begin to separate the disc into two halves. The secondary cracks, i.e. cracks parallel to the loading diameter, continue to crack in vertical direction and bifurcate in any possible direction (Fig. 3 from $55 \mu \mathbf{S}$ to $168 \mu \mathrm{S}$ ). At the same time, more cracks are produced on the contact area of loading palates and disc.

According to experiments (Fig.5) conducted by Malan et al.[16], typical fracture propagation in Brazilian disc test includes primary tensile cracking along the loading diameter, secondary cracking from the sides parallel to the primary cracks, and tertiary cracking due to shear failure at the contact areas of plates and disc. Comparing the modelling results with the experiments results, the Hybrid finite-discrete element method well reproduces the primary cracks and the secondary cracks. Probably due to the relatively big mesh size, the tertiary cracks near the plates mix with secondary cracks near the plates (Fig.3 at $168 \mu$ s). 


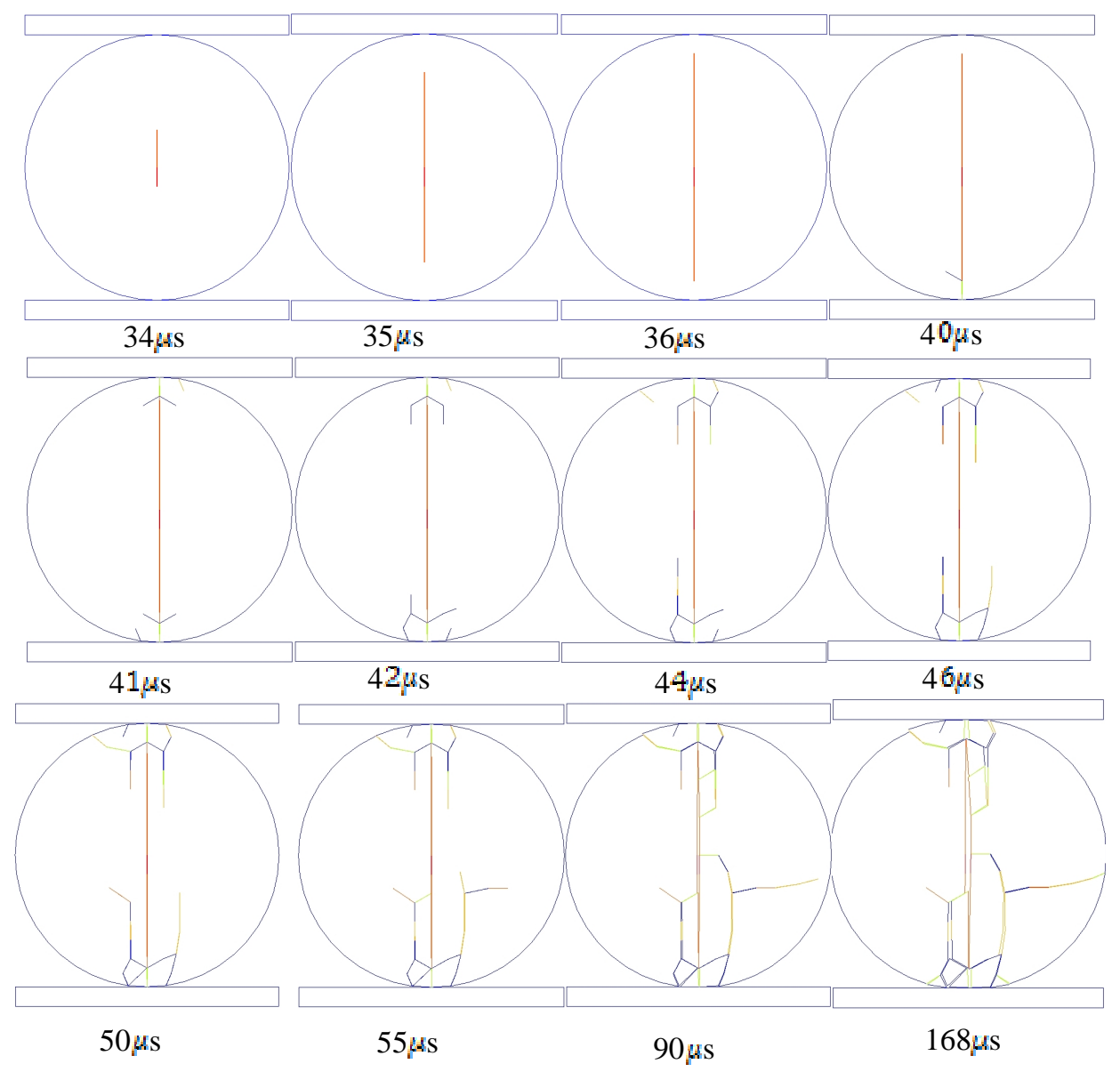

Fig .4 Fracture initiation and propagation of rock under constant displacement increment of $1 \mathrm{~m} / \mathrm{s}$

According to the researches by [17] and [18], a typical failure pattern of Brazilian disc test under static loading include tensile failure along the loading diameter and shear failure at bottom and top contact place as shown in Fig.6a. According to the numerical process in Fig. 4 where the red represents the tensile failure while the blue shear failure, the numerical result is in accordance with the research results in terms of failure types. Meanwhile, according to the experimental results in various materials as shown in Fig.6b [19], cracks generally occur at the loading points and/or from the center of the diameter propagating to the loading points. Therefore, the numerical result of Brazilian disc text modelled by Y-2D/3D IDE agrees well with the experimental results in Fig.5b.
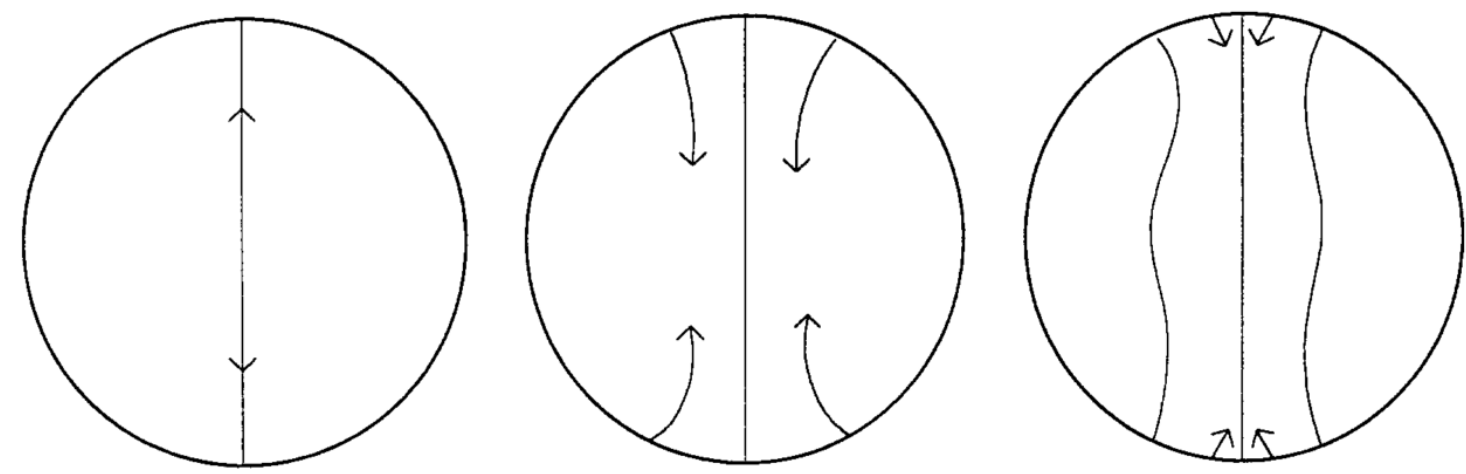

a) Primary tensile cracking b) secondary cracking on both sides of loading diameter c) tertiary cracking due to shear failure near platens

Fig.5 Typical fracture propagation in Brazilian Disc test[16, 20] 


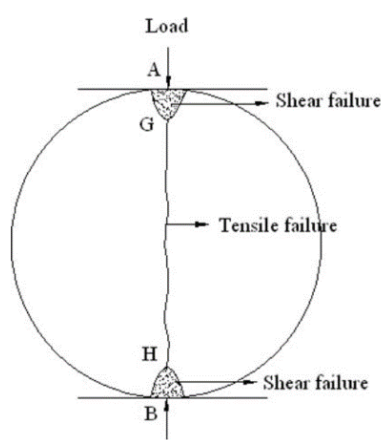

a) Typical failur types $[17,18]$

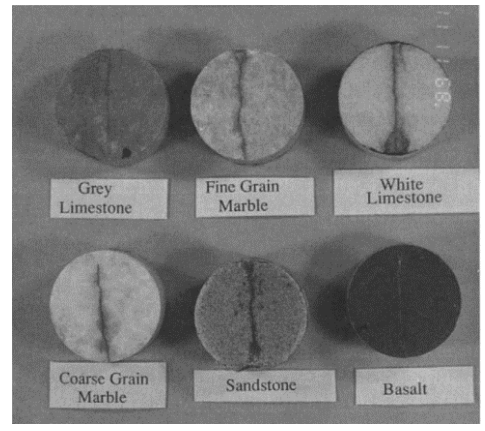

b) Exprimental results of Brazilian disc test in various materials[19] Fig.6

\section{Conclusions}

A Hybrid finite-discrete element method is introduced and the combined single and smeared crack model is implemented to the proposed method to simulate the transition from continuum to discontinuum through fracture and fragmentation. Brazilian disc test is modelled using Hybrid finite-discrete element method to calibrate the proposed method. The modelled temporal and distribution of the stress are captured and the fracture initiation and propagation of rock are modeled. The stress distribution and fracture pattern are compared with numerical and laboratory tests and agree well with them.

It is concluded that the modelled results keep great accordance with the experimental and theoretical research in terms of stress initiation and propagation, failure process and failure pattern. Therefore, Hybrid finite-discrete element method is a useful tool in modelling the fracture and fragmentation of brittle materials. Moreover, Y-2D/3D IDE shows its great abilities in modelling the fracture initiation and propagation.

\section{Acknowledgements}

This work was partly supported by a visiting $\mathrm{PhD}$ scholarship provided by China Scholarship Council, the National Natural Science Foundation of China ( 51208036 ) and the Fundamental Research Funds for the Central University (FRF-TP-14-075A2), which are greatly appreciated.

Specially, the first author would like to thank Dr. Hongyuan Liu, who supervised him to study the Hybrid finite-discrete element method in university of Tasmania.

\section{References}

[1] Liu, H., S. Kou, P.-A. Lindqvist, and C. Tang, Numerical modelling of the heterogeneous rock fracture process using various test techniques. Rock mechanics and rock engineering, 2007. 40(2): p. 107-144.

[2] Mahabadi, O., B. Cottrell, and G. Grasselli, An example of realistic modelling of rock dynamics problems: FEM/DEM simulation of dynamic Brazilian test on Barre granite. Rock mechanics and rock engineering, 2010. 43(6): p. 707-716.

[3] Stefanizzi, S., G. Barla, P. Kaiser, and G. Grasselli. Numerical modeling of standard rock mechanics laboratory tests using a finite/discrete element approach. in Proceedings of the 3rd CANUS Rock Mechanics Symposium, Toronto. 2009.

[4] Zhu, W. and C. Tang, Numerical simulation of Brazilian disk rock failure under static and dynamic loading. International Journal of Rock Mechanics and Mining Sciences, 2006. 43(2): p. 236-252. 
[5] Morris, J., M. Rubin, S. Blair, L. Glenn, and F. Heuze, Simulations of underground structures subjected to dynamic loading using the distinct element method. Engineering computations, 2004. 21(2/3/4): p. 384-408.

[6] Liu, H., Y. Kang, and P. Lin, Hybrid finite-discrete element modeling of geomaterials fracture and fragment muck-piling. International Journal of Geotechnical Engineering, 2013.

[7] Jing, L. and J. Hudson, Numerical methods in rock mechanics. International Journal of Rock Mechanics and Mining Sciences, 2002. 39(4): p. 409-427.

[8] Jing, L., A review of techniques, advances and outstanding issues in numerical modelling for rock mechanics and rock engineering. International Journal of Rock Mechanics and Mining Sciences, 2003. 40(3): p. 283-353.

[9] Latham, J.-P., A. Munjiza, X. Garcia, J. Xiang, and R. Guises, Three-dimensional particle shape acquisition and use of shape library for DEM and FEM/DEM simulation. Minerals Engineering, 2008. 21(11): p. 797-805.

[10] Mohammadi, S., Discontinuum mechanics: Using finite and discrete elements. 2003: WIT press Southampton.

[11] Morin, M.A. and F. Ficarazzo, Monte Carlo simulation as a tool to predict blasting fragmentation based on the Kuz-Ram model. Computers \& geosciences, 2006. 32(3): p. 352-359.

[12] Munjiza, A., The Combined Finite-Discrete Element Method. 2004: Wiley Online Library.

[13] An, H. and H. Liu. Hybrid finite-discrete element modelling of dynamic fracture of rock and resultant fragment arching by rock blast. in 8th Annual Asian Rock Symposium. 2014.

[14] Munjiza, A., K. Andrews, and J. White, Combined single and smeared crack model in combined finite - discrete element analysis. International Journal for Numerical Methods in Engineering, 1999. 44(1): p. 41-57.

[15] Zhang, Z., Laboratory studies of dynamic rock fracture and in-situ measurements of cutter forces for a boring machine. 2001.

[16] Malan, D., J. Napier, and B. Watson. Propagation of fractures from an interface in a Brazilian test specimen. in International Journal of Rock Mechanics and Mining Sciences \& Geomechanics Abstracts. 1994. Elsevier.

[17] Hobbs, D. The tensile strength of rocks. in International Journal of Rock Mechanics and Mining Sciences \& Geomechanics Abstracts. 1964. Elsevier.

[18] Li, D. and L.N.Y. Wong, The Brazilian disc test for rock mechanics applications: review and new insights. Rock mechanics and rock engineering, 2013. 46(2): p. 269-287.

[19] Guo, H., N. Aziz, and L. Schmidt, Rock fracture-toughness determination by the Brazilian test. Engineering Geology, 1993. 33(3): p. 177-188.

[20] Colback, P. An analysis of brittle fracture initiation and propagation in the Brazilian test. in 1st ISRM Congress. 1966. International Society for Rock Mechanics. 